Historic, Archive Document

Do not assume content reflects current scientific knowledge, policies, or practices. 

2,29

\section{SBASON 1916. WHOLESALE PRICE LIST CROP 1915. Garden, Field and Flower Seeds}

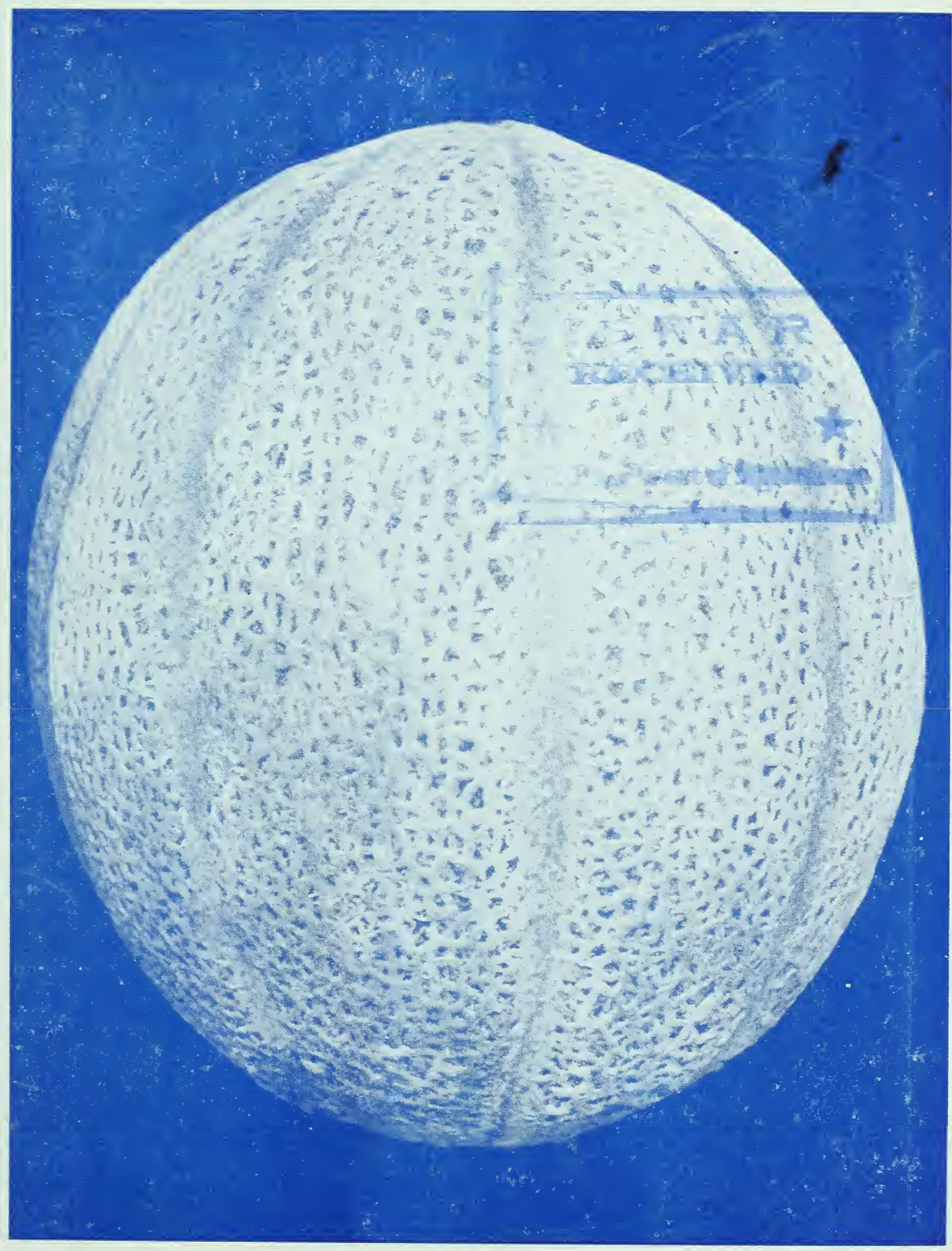

\section{The Mangelsdorf Bros. Co., Atchison, Kansas.}




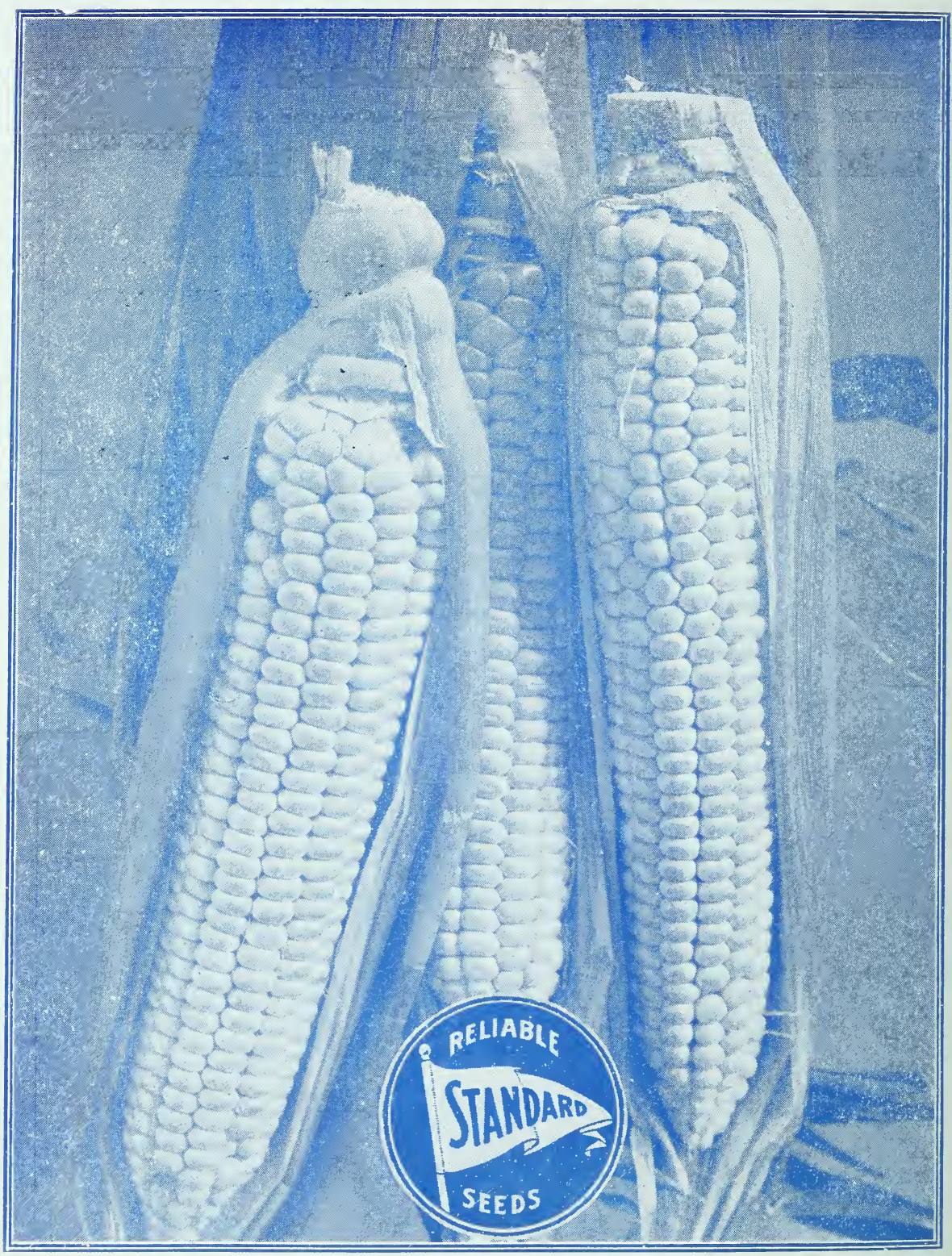

CORN. EARLY EVERGREEN. 


\section{TO THE TRADE}

W

E HEREWITH present our Wholesale Price List of fresh and carefully selected GARDEN, FLOWER and AGRICULTURAL SEEDS. Our seeds are grown by the most careful growers from pure and selected stocks, and we use every care to have all seeds we send out just as represented, but it must be understood that we do not in any way warrant them, nor will we be responsible for any failure thereof. At the same time we are always ready to consider any well-founded complaints.

We will not be undersold byFanyire- $E E$ i $V E$ liable house on seeds of like quality. Quality is always our firse consider $A P R 111916$
ation. Cheap seeds are dea as gifte

NOTICE-We sell 10 pounds at the 100 pound rate, one-fourth bushel at bushel rates, and one-fourth pound atorgoniodand tsostembitienBotany a smaller quantity is ordered a small advanoe will be charged.

All prices are subject to market fluctuations.

All seamless bags will be charged extra at cost price and are not returnable.

\section{TERMS}

Grass, Clover, and all Field and Agricultural Seeds, Onion Sets, Potatoes and Sweet Potatoes, are sold for NET CASH only.

All accounts of Vegetable Seeds are sold on terms of 60 days, 2 per cent discount 10 days' payment.

Overdue accounts subject to SIGHT DRAFT, with exchange, without notice.

Satisfactory references required from new customers.

All prices subject to change without notice.

\section{ADVERTISING MATTER}

Fine lithographed "HANGERS" for the window ; "LABELS" for Seed Boxes and Jars, and Retail Catalogues for distribution to customers furnished free with every order for Bulk Garden Seeds.

\section{WE BUY}

He are always in the market to buy Timothy, Clover, Kaffir, Sorghum, Villet, Pop Corn, etc., and are ready to offer full market price. Pags for mailing samples sent on request.

\section{The Mangelsdorf Bros. Co.}




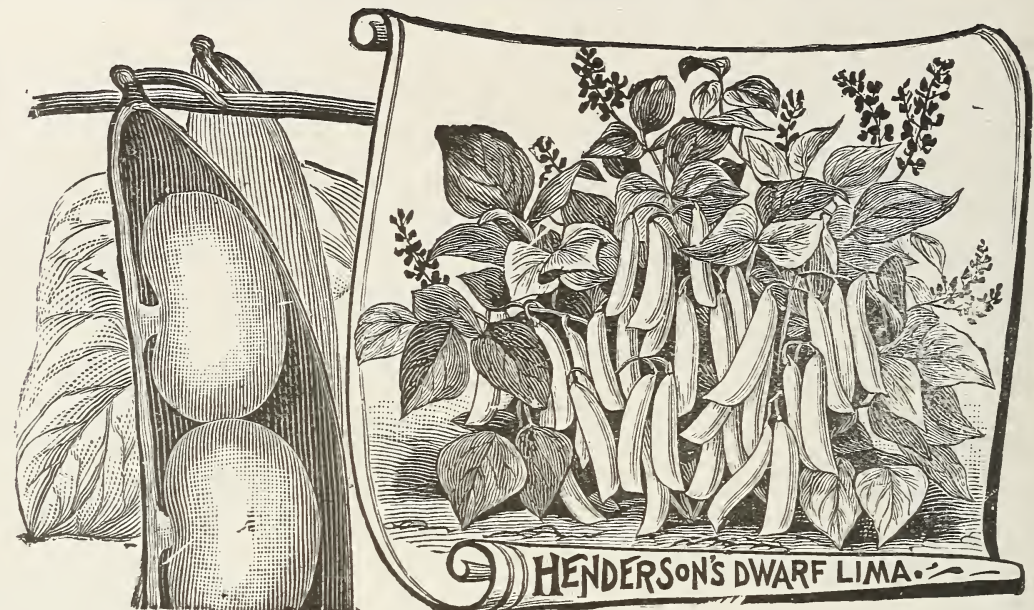

ASPARAGUS

Conover's Colossal.

Per lb

Columbian Mammoth White.................... 35

$10 \mathrm{lbs}$.

Per lb. $\$ 0.22$ .30

\section{BEANS-Dwarf or Bush}

Green Podded Sorts

Per bu.

IMPROVED RED VALENTINE, very early and hardy..........

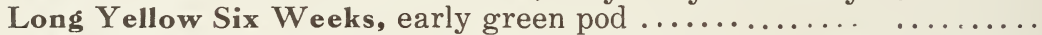

STRINGLESS GREEN POD, excellent flavor and prolific . . . . . . .

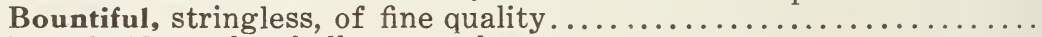

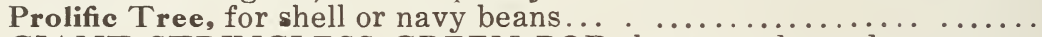

GIANT STRINGLESS GREEN POD, large tender pods.........

\section{BEANS-Wax or Yellow Podded Sorts}

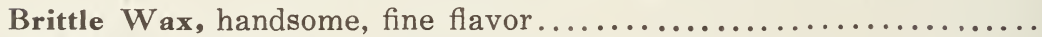

Challenge Black Wax, favorite market gardeners' sort...........

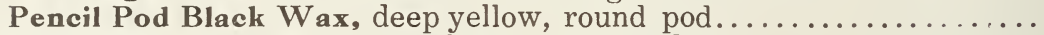

PROLIFIC BLACK WAX, large meaty pods.....

IMPROVED RUST PROOF GOLDEN WAX, prolific and rust proof

WARDWELL'S KIDNEY WAX, broad and handsome pod........

Davis Kidney Wax, white seeded, long, beautiful pod.............

\section{Pole or Running Sorts}

Kentucky Wonder Wax, rich golden yellow pods ............ 7.50

KENTUCKY WONDER, the best of all pole beans ........... 6.00

Lazy Wife, a favorite sort ......................... 7.00

Cut Short or Corn Hill, excellent for planting among corn ... . . 6.50

\section{Limas}

Ford's Mammoth Pod Pole Lima, vigorous grower, large pods...... 6.50

King of the Garden, pole, pods $5-6$ inches long ................ 6.50

Henderson's Bush Lima, height one foot, very productive ......... 7.00

Burpee's Bush Lima, dwarf, large pods.............................. 70

All Beans are sold 60 lbs. to the bushel, except Pole and Dwarf Limas, which are $56 \mathrm{lbs}$. to the bushel. 


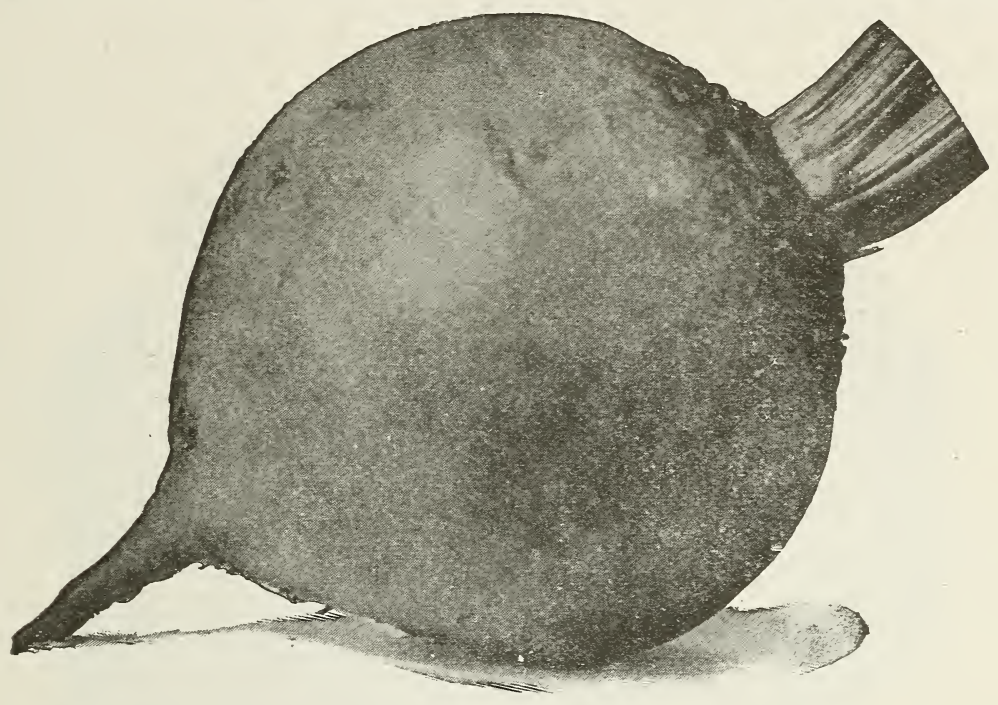

Beet. Crimson Globe.

\section{BEETS-Table Sorts}

$10 \mathrm{lbs}$.

Per lb. Per lb.

CRIMSON GLOBE, handsome form, deep crimson........\$0.60 \$0.50

CROSBY'S EGYPTIAN, the earliest, deep red, small tops. .60

Early Egyptian Turnip, of quick maturity, flat shape...... . 60

Eclipse, globe shape, dark flesh, very sweet.............. 60

Edman's Improved Blood Turnip, a popular turnip shaped beet

EARLY BLOOD TUR NIP, the standard early.............. .60

Detroit Dark Red, an excellent market sort... ......... . .60

Half Long Blood, smooth and thick.................. .60

Long Dark Red, long, tapering, good keeper.......... .60

.50

.50

.50

.50

.50

.50

.30

.50

\section{BEETS - For Sugar or Stock Feeding Mangel Wurzels}

Mammoth Long Red, enormously productive............ \$0.25

Golden Tankard, the best yellow sort .................... 25

Great Dane Half Sugar Mangel, excellent for stock feed .... .25

Sugar Beets

Vilmorin's Improved White $\ldots \ldots \ldots \ldots \ldots \ldots \ldots \ldots \ldots \ldots . \ldots \ldots$

Kleine Wansleben, very rich in sugar................... $\quad .25$

\section{CAULIFLOWER}

Finest Danish Grown Per oz. Per lb.

EARLY SNOWBALL, best for general use ........... $\$ 1.50 \quad \$ 20.00$

Extra Early Paris, very early, reliable header ............ .50 6.00 


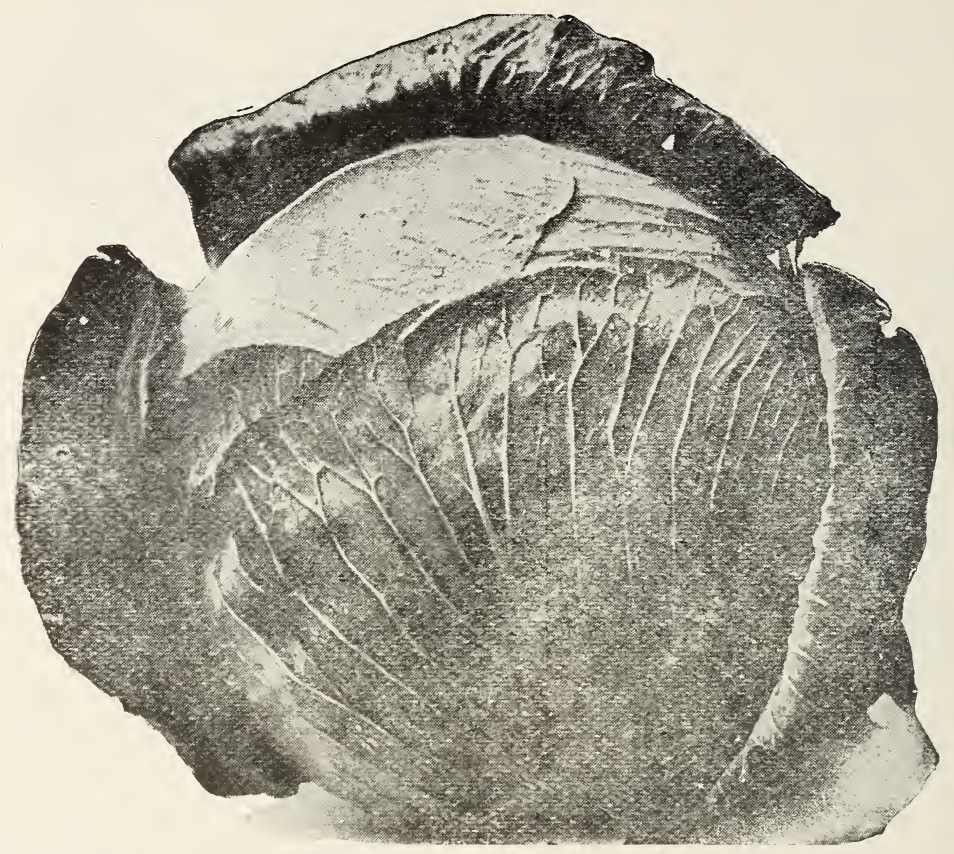

Cabbage. Premium Flat Dutch.

\section{CABBAGE-Finest American Grown $10 \mathrm{lbs}$.}

\section{Early Pointed Heads. Per lb.}

Extra Early Express, the earliest sort.............. $\$ 1.00$

Early York, a favorite variety in many sections.......... 1.n0

EARLY JERSEY WAKEFIELD, the best early.......... 1.25

Charleston or Large Wakefield, fine market gardeners' sort. 1.25

EARLY WINNINGSTADT, hardy and sure header ..... 1.25

\section{Early Flat Heads.}

Early Spring, earliest flat head .................. 1.10

EARLY ALLHEAD, the best second early.............. 1.10 .

All Seasons, excellent sort, firm heads ................ 1.10

Henderson's Early Summer, matures early, large flat heads. 1.10

EARLY FLAT DUTCH, the favorite second early........ 1.10

\section{Late or Winter Sorts.}

PREMIUM FLAT DUTCH, finest strain selected seed.... 1.10 Hollander, hardy and solid ........................ 150 Large Late Drumhead, good keeper, solid heads.......... 1.10 SUREHEAD, finest strain, reliable header, fine quality.... 1.10

\section{Red Cabbage}

Mammoth Red Rock, the best late sort .............. $1.50 \quad 1.25$

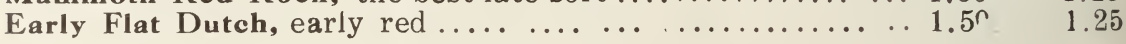




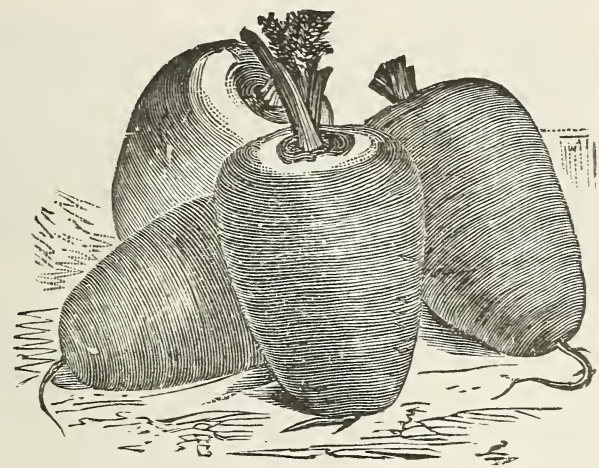

Carrot. Early Oxheart.

\section{CARROT}

$10 \mathrm{lbs}$. Per lb. Per lb. $\$ 0.45$

\section{CELERY}

\section{Our Stocks are the Best Obtainable.}

WHITE PLUME, extra fine, pure white and brittle......\$1.50 GOLDEN SELF BLANCHING, dwarf, compact, thick solid

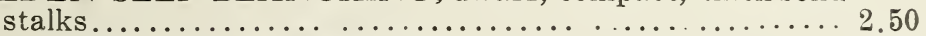
Giant Paschal, the best winter sort...................... 1.50 Celeriac or Turnip Rooted, ....................... 1.50

\section{COLLARDS}

Southern Giant.... $\$ 0.40$

\section{GRESS OR PEPPER GRASS}

Dwarf Fine Curled.

\section{SWEET CORN}

Prices Quoted Are Per Measured Bushel, Shelled. Per bu.

Golden Bantam, small, sweet, yellow ear ................ \$4 50

Mammoth White Cory, excellent quality, early ...............

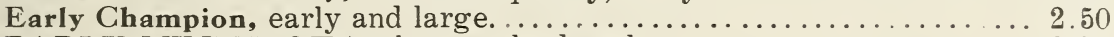

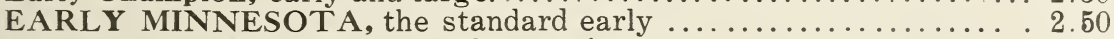

STOWELL'S EVERGREEN, best main crop ............. . 2.50

COUNTRY GENTLEMAN, very productive, small sweet grains . . 350

Late Mammoth, very large, late ........................... 50

EARLY EVERGREEN, two weeks earlier than Stowell's Evergreen 2.5')

New White Mexican, same as Black Mexican except in color...... 350

Black Mexican, of quick maturity, sweet and luscious ........ 2.75

Extra Early Adams, very popular for first roasting ears .......... 2.00

All Sweet Corn sold per measured bu. No standardweight for Sweet Corn

$10 \mathrm{lbs}$

CORN SALAD OR FETTICUS Per lb. Perlb.

Improved Broad Leaved......................\$0.50 \$ \$0.40 


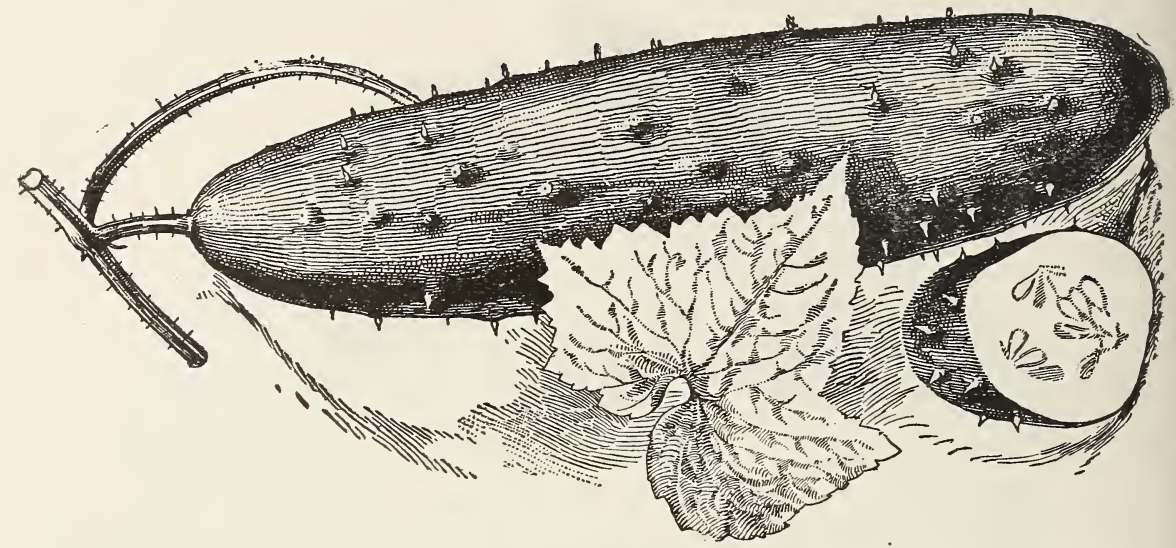

Cucumber. Chicago Pickling.

\section{GUCUMBER}

$10 \mathrm{bs}$

Early Frame or Short Green, early and productive ........ $\$ 0.50 \$ 0.40$

Early Cluster, remarkably free bearing. . ............. .50 .40

IMPROVED WHITE SPINE, market gardeners' strain ... $\quad .50 \quad .40$

Emerald, extra choice, bright green.................. $\quad .50 \quad .40$

Japanese Climbing . . . . . . . . . . . . . . . . . . . . . . . . .75

Gherkin, or Burr........... ................ . $55 \quad .70$

Boston Pickle, favorite pickling sort ............... $.50 \quad 40$

IMPROVED LONG GREEN, quality unsurpassed........ $.55 \quad .45$

DAVIS PERFEGT, best for forcing ................. .50 $\quad .40$

Chicago Pickling, small, fine form and color............ $.50 \quad .40$

ENDIVE

Green Curled.............................. $\$ 0.70$

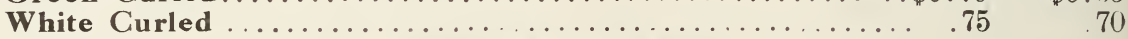

\section{EGG PLANT}

Improved New York Purple ................\$3.50

BLACK BEAUTY......................... $4.00 \quad 3.75$

\section{KALE}

Tall Green Curled....................... $\$ 0.50$

Dwarf Green Curled.......................... .50

\section{KOHL RABI}

Early Purple Vienna

Early White Vienna

\section{LEEK}

Monstrous Carentan, choice 


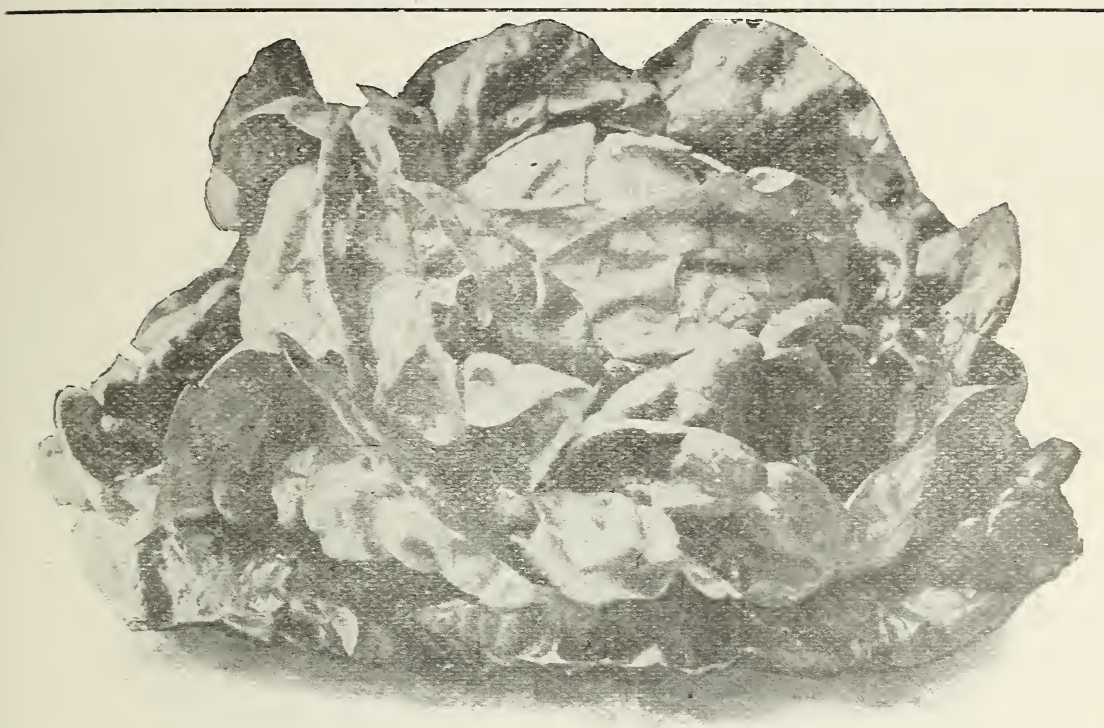

Lettuce. Big Boston.

\section{LETTUCE}

\section{EARLY CURLED SORTS}

$10 \mathrm{lbs}$.

Per lb. Per lb.

SIMPSON'S EARLY CURLED, fine, loose, curled heads... \$0.45

Simpson's Black Seed, fine for summer............... .40

GRAND RAPIDS FORCING, popular market sort ... . . . .45

PRIZEHEAD, the standard sort.................... 45

\section{HEADING SORTS}

HANSON, a splendid summer variety .............\$0.50

Denver Market, large, solid heads ................... . $\quad .45$

Iceberg, excellent, crisp and tender........... . .50

Philadelphia Butter, round, thick leaves, rich flavor....... .55

BIG BOSTON, large, firm heads, resemble cabbage.. ... .50

Deacon, very solid, early........................... 50

May King, globe shaped, mild and tender $\ldots \ldots \ldots \ldots \ldots . .50$

$\$ 0.40$

40

$\$ 0.40$

\section{MUSKMELON}

BURRELL GEM, thick pink flesh, small seed cavity.......\$0.60

Banana, long yellow sort............................ 60

EXTRA EARLY HACKENSACK, early and of good size.. $\quad .60$

Netted Gem, selected strain .......................... .60

ROCKY FORD, genuire oval strain..................... 60

Paul Rose, new, yellow flesh, fine. .................. .60

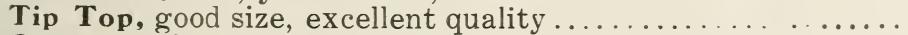

Osage, market gardeners' stock ..... $\quad \ldots \ldots \ldots \ldots \ldots \ldots . .60$

Early Green Nutmeg, green flesh, small, extra early ... $\quad .60$

Bay View, large, fine flavor, green flesh $\ldots \ldots \ldots \ldots, \quad 60$

Improved Canteloupe, the best large variety............. $\quad .60$

HOODOO, a greatly improved Rocky Ford type ............ .60

LARGE HACKENSACK, splendid main crop sort......... .60 


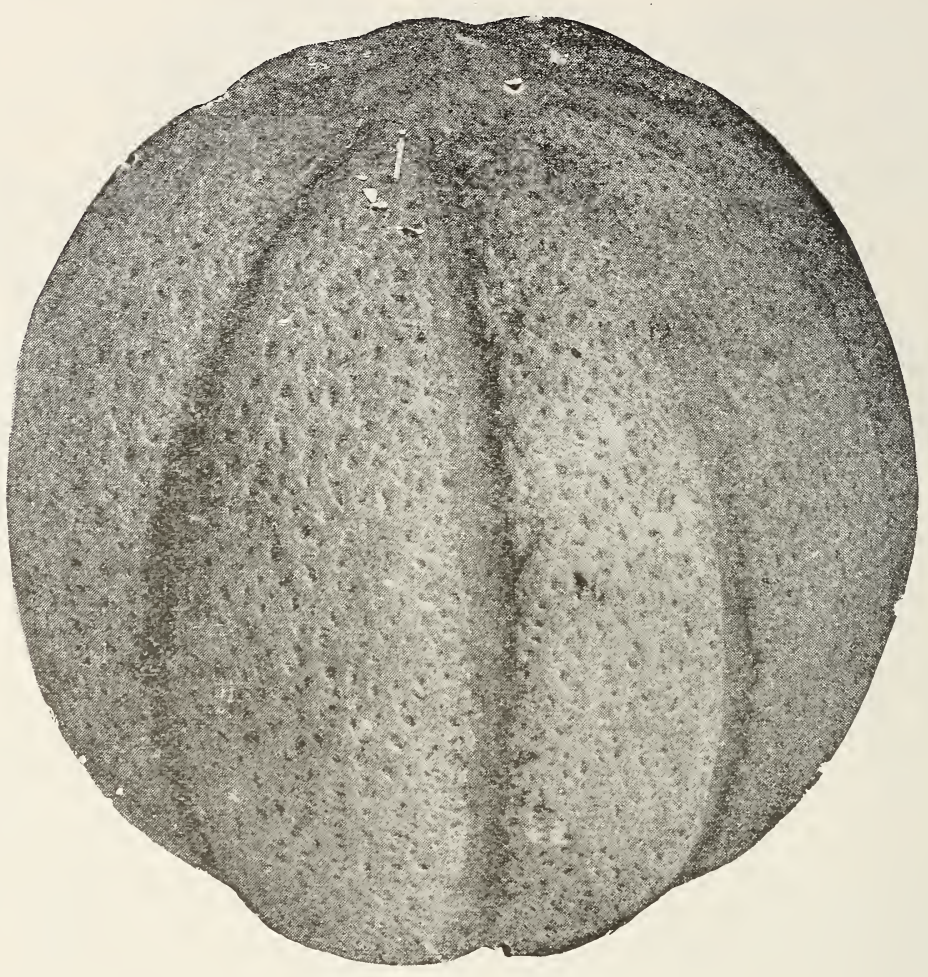

Muskmelon Hoodoo.

\section{WATERMELON}

$10 \mathrm{lbs}$.

Per lb. Per lb.

HARRIS’ EARLIEST, very early, good size, striped variety. $\$ 0.35$

Ice Cream, early, medium size, oblong shape.......... . .35

Phinney's Early, the earliest small .................. . 35

Gray Monarch or Long Light Icing ............... .35

Triumph, new, mammoth size...................... . .35

SWEETHEART, fine shipping sort, large size, pale green rind .35

Cuban Queen, an old favorite.................... . 35

Dark Icing, firm, thin rind, good shipper.............. .35

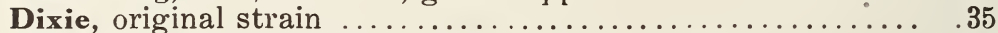

FLORIDA FAVORITE, oblong, striped, fine flavor ...... .35

KOLB'S GEM, a popular striped sort of large size ....... .35

KLECKELY'S SWEET, superb sort, dark skin, very thin rind $\quad .50$

McIver's Wonderful Sugar, very sweet $\ldots \ldots \ldots \ldots \ldots \ldots \ldots \ldots$

GEORGIA RATTLESNAKE, long striped........... . 35

Mountain Sweet, excellerit old standard variety .......... 35

ALABAMA SWEET, fine shipping melon.............. .35

TOM WATSON. a new melon of finest quality. ........ .45

HALBERT HONEY, quality unequaled ...............5

Citron, Red Seeded ................................. ${ }_{4}^{45}$ 


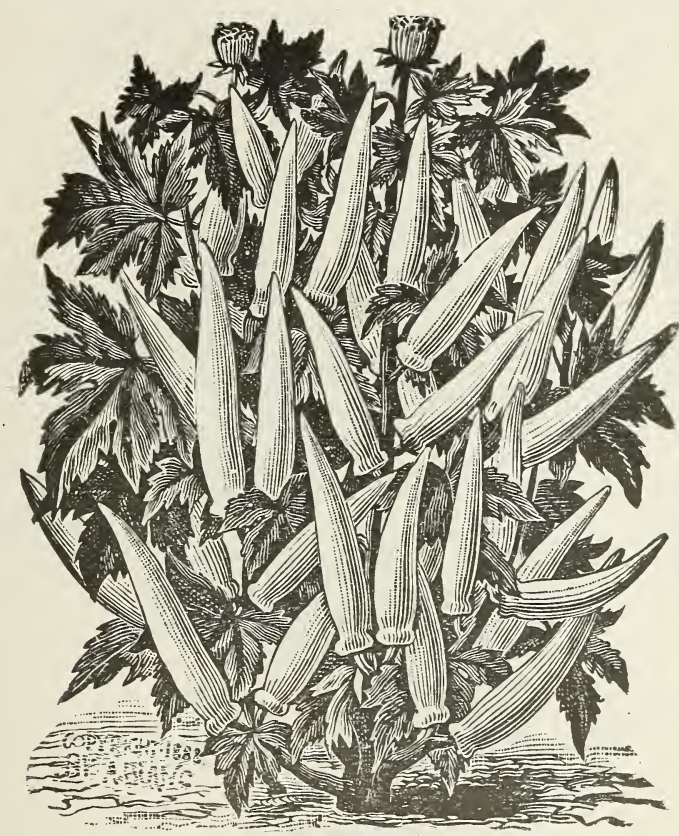

Okra. White Velvet.

\section{MUSTARD}

$10 \mathrm{lbs}$. Per lb. Per lb.

Southern Giant, a popular southern sort, large curled leaves.\$0.30 $\$ 0.25$ Ostrich Plume, beautiful densely curled leaves, fine flavor... . 30 .25

White or English, for salads or flavoring ............. .25 $\quad .20$

\section{ONION-White}

Our Onion Seed is Finest American Stock

Earliest White Queen, small pickling sort ............2.50 2.25

Southport White Globe, finest strain, pure white... . . $\quad 2.50 \quad 2.25$

WHITE PORTUGAL OR SILVER SKIN, large, early, mild flavored....................... 2.25

\section{YELLOW}

YELLOW GLOBE, DANVER'S, standard yellow ........ 1.75

Prizetaker, largest yellow, immense yielder .......... . 1.50

Australian Brown, of sterling merit ................. 1.50

Australian Yellow Globe, brownish yellow color ......... 1.50

RED

Extra Early Red Flat, very early .................. 2. . . . . .

RED WEATHERSFIELD, standard red for all localities . . 2.00

Southport Red Globe, best strain ................ 2.00 


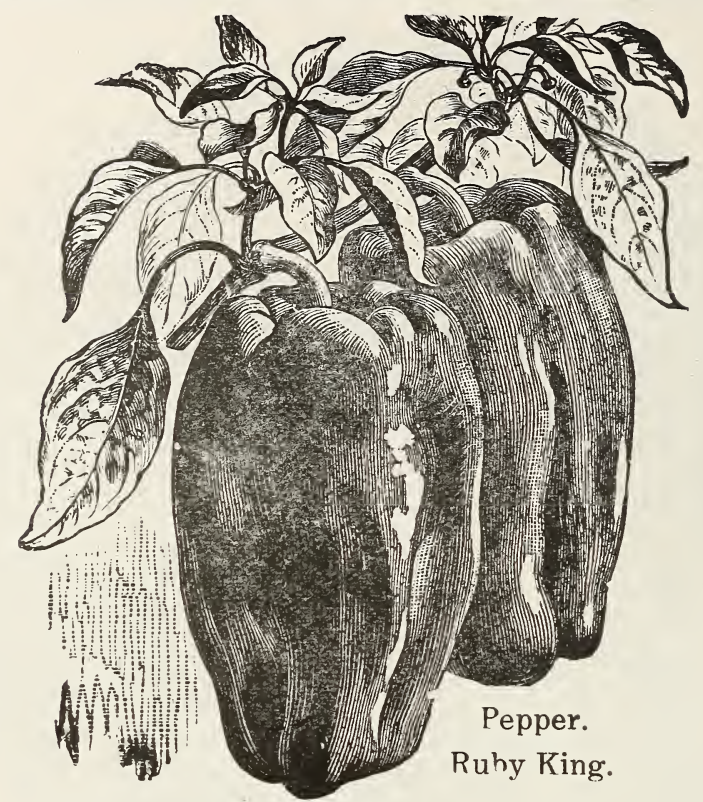

OKRA

Per lb.

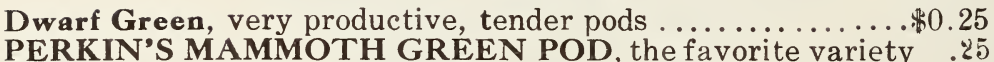

White Velvet, long, smooth pods, light color........... .25

\section{PARSLEY}

Extra Triple Curled or Emerald ................... . .5

Dark Moss Curled, densely curled ................. . .50

\section{PARSNIP}

Long Smooth................................ . 30

HOLLOW GROWN, choicest stock, thick shoulder ........ .30

\section{PEPPER}

SWEET MOUNTAIN, large, mild ................. 2.50

Bell or Bull Nose, large red........................ 2.00

RUBY KING, extra large and mild................... 2.00

Long Red Cayenne, hot and pungent............. 1.75

GHINESE GIANT, immense size, mild flavor ......... 3.50

\section{PUMPKIN}

CONNECTICUT FIELD, very proliffc.............. .25

Quaker Pie, fine grained, rich flavor $\ldots \ldots \ldots \ldots \ldots \ldots \ldots \ldots . .50$

Winter Luxurv, good keeper ......................... $\quad .50$

SMALL SUGAR, very sweet and mild .............. .50

LARGE CHEESE OR KY. FIELD, a favorite field sort . . . $\quad .20$

Tennessee Sweet Potato, striped, pear shaped variety...... $\quad .50$

Gushaw or Crookneck, productive and good keeping qualities .75

KING OF MAMMOTH, largest sort ................60
10 lbs.

Per lb.

$\$ 0.20$

.20

.20

.40

.25

.25

2.25

1.75

1.75

1. 50

2.25 


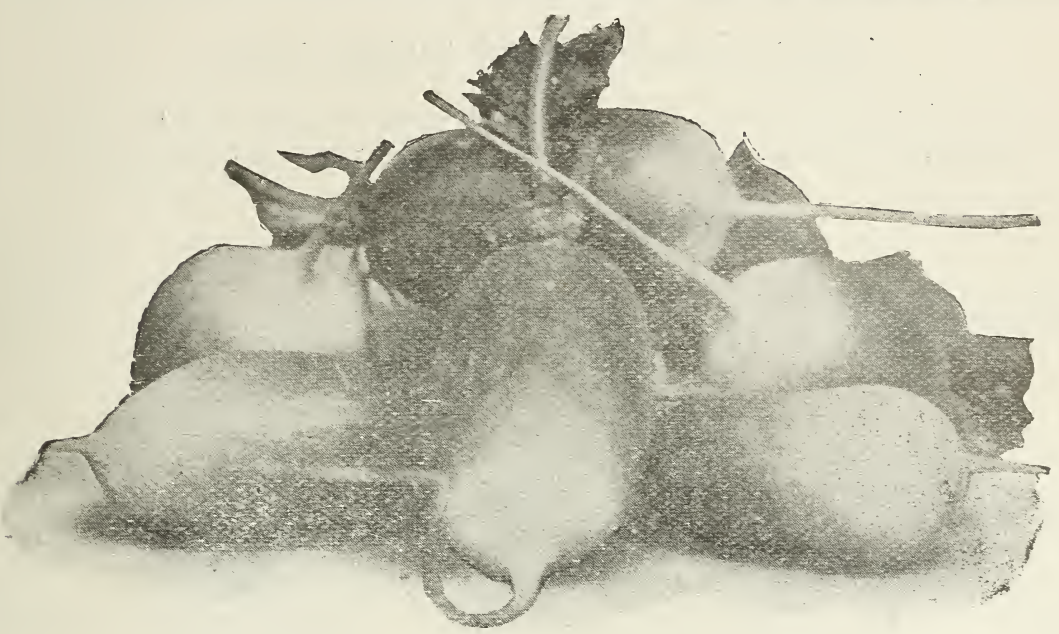

Radish. French Breakfast.

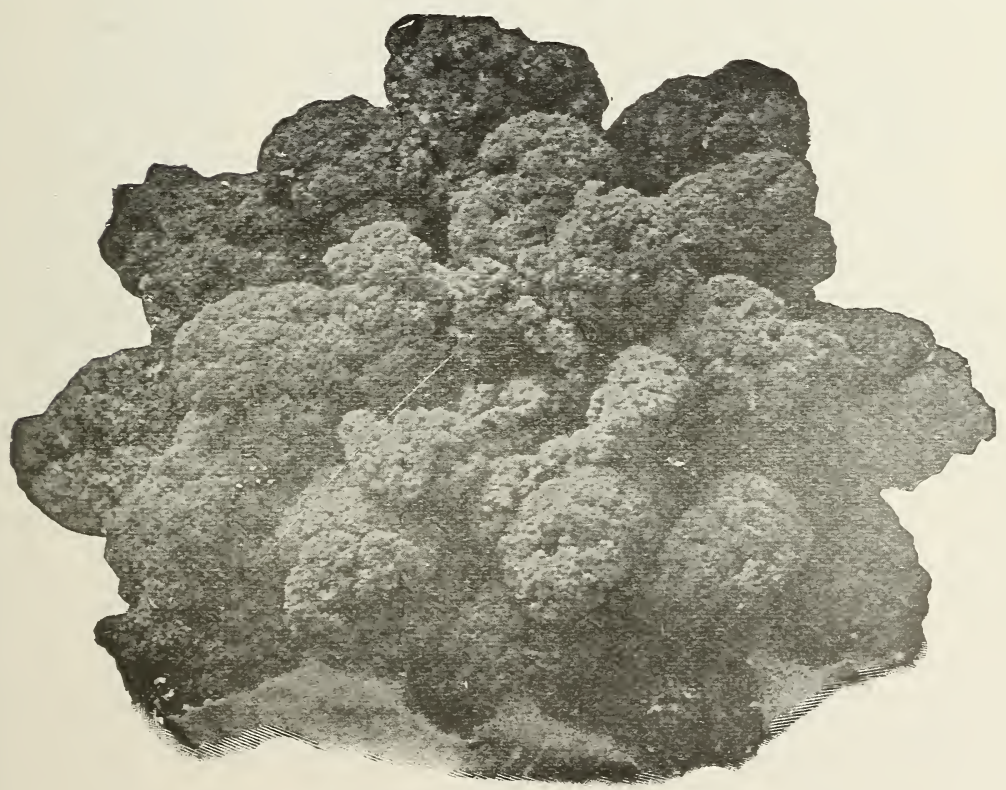

Parsley. Emerald. 


\section{PEAS-Choice Hand Picked Stock}

* Denotes wrinkled peas; these are sold at $56 \mathrm{lbs}$. per bushel. Varieties not marked are smooth seeded sorts and are sold at $60 \mathrm{lbs}$. per bushel.

\section{EXTRA EARLY SORTS}

Per bu.

Alaska, 2 ft., extra early, very prolific .................\$4.50 FIRST AND BEST, $2 \mathrm{ft} .$, the standard early .............. 4.50

\section{EARLY DWARF SORTS}

*Thos. Laxton, $3 \mathrm{ft}$., an extra fine sort ..................\$n. 00 *Nott's Excelsior, 15 inches, one of the best.............. 5.50 *AMERICAN WONDER, 12 inches, sweet and fine flavored ..... 5.50 *CARTER'S PREMIUM GEM, 15 inches, favorite dwarf . ....... 5.25 Tom Thumb, 12 inches, early and prolific ................. 50 *GRADUS, 3' ft., extra early, enormous pods ............... 5.00 *SUTTON'S EXCELSIOR, 12 inches, extra quality, best dwarf ... 5.75

\section{LATER SORTS}

*TELEPHONE, $3 \frac{1}{2} \mathrm{ft}$. . best tall sort...................

McLean's Advancer, '2 feet, handsome pods, vigorous growth .....4.75

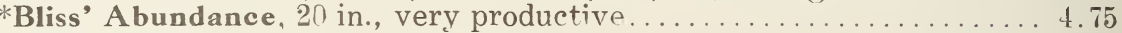

*EVERBEARING, 2 ft., unexcelled...................4.50

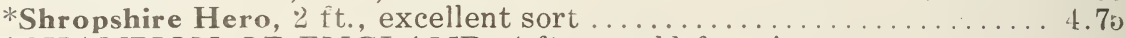

*CHAMPION OF ENGLAND, $4 \mathrm{ft}$., an old favorite...........4.50

White Marrowfat, hardy and productive ............... . 4.50

*Horsford's Market Garden, 2 ft., bears profusely . . . . . . . . . 4.75

*Stratagem, 2 ft., heavy cropper, excellent quality ................

\section{RADISH}

$10 \mathrm{lbs}$.

Per lb. Per lb.

Non Plus Ultra

$\$ 0.40 \$ 0.35$

Earliest White Turnip Forcing

Earliest White Olive Shaped.

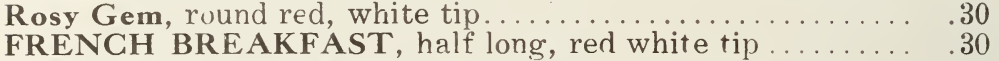

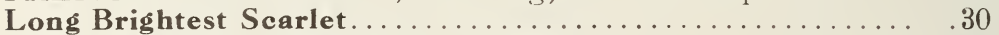

EARLY LONG SCARLET SHORT TOP .......... . 30

Early White Summer Turnip...................... . 30

ICICLE, earliest long white................... 31

EARLY SCARLET TURNIP, favorite round red . . . . . . . 30

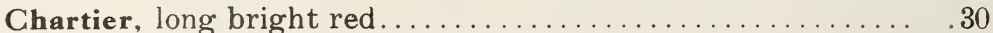

White Vienna, long white early................. 30

WHITE STRASBURG, good for summer use........... .30

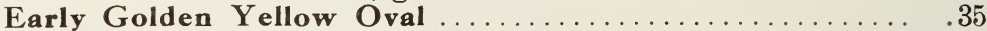

Long Black Spanish, winter.................. 40

Rose China, winter ......................... 40

CRIMSON GIANT, largest size, crisp and teuder ....... .40

Chinese or Celestial, long white winter ..............40

Half Long Deep Scarlet......................... 40 


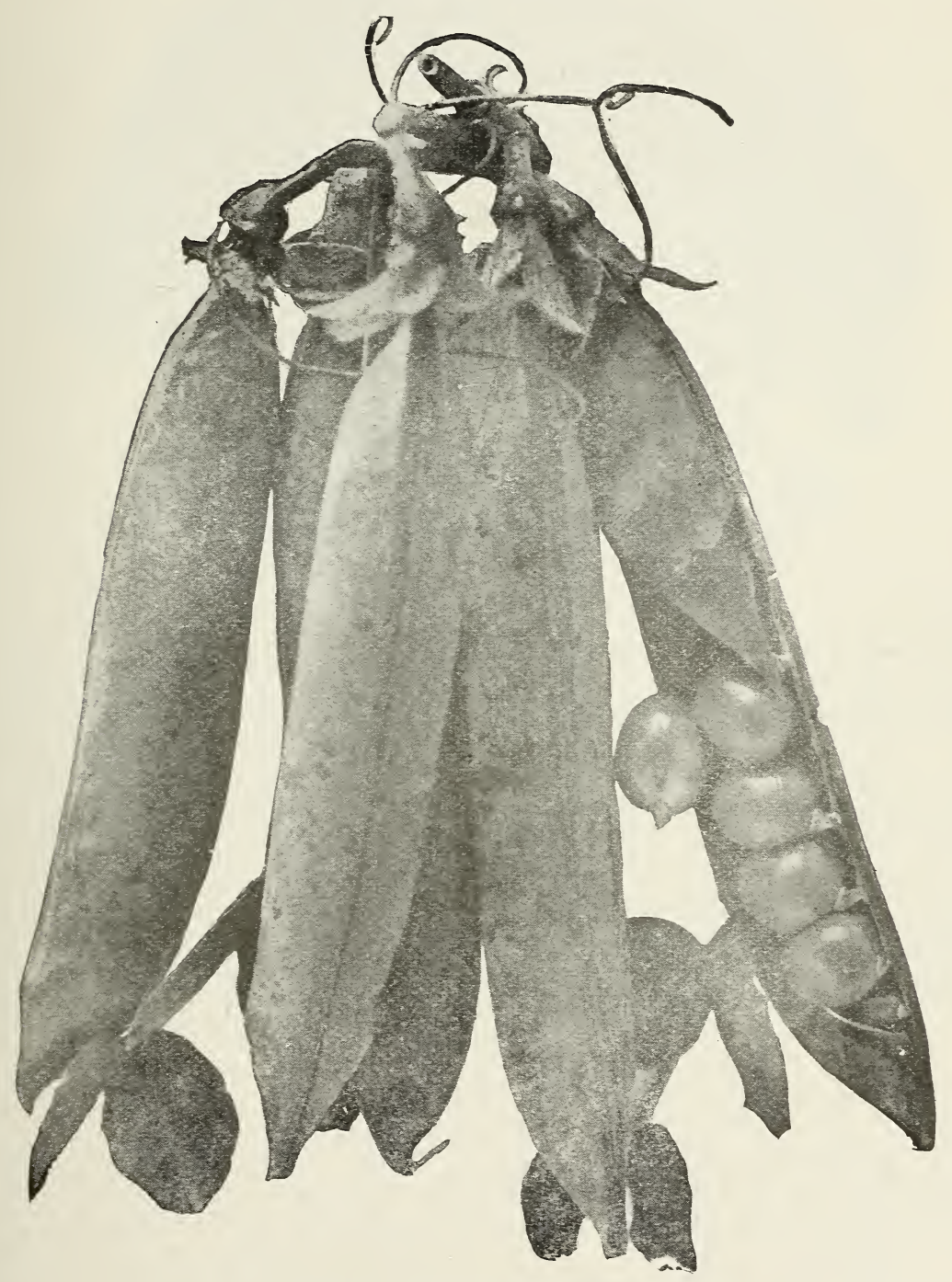

Peas. Sutton's Excelsior. 


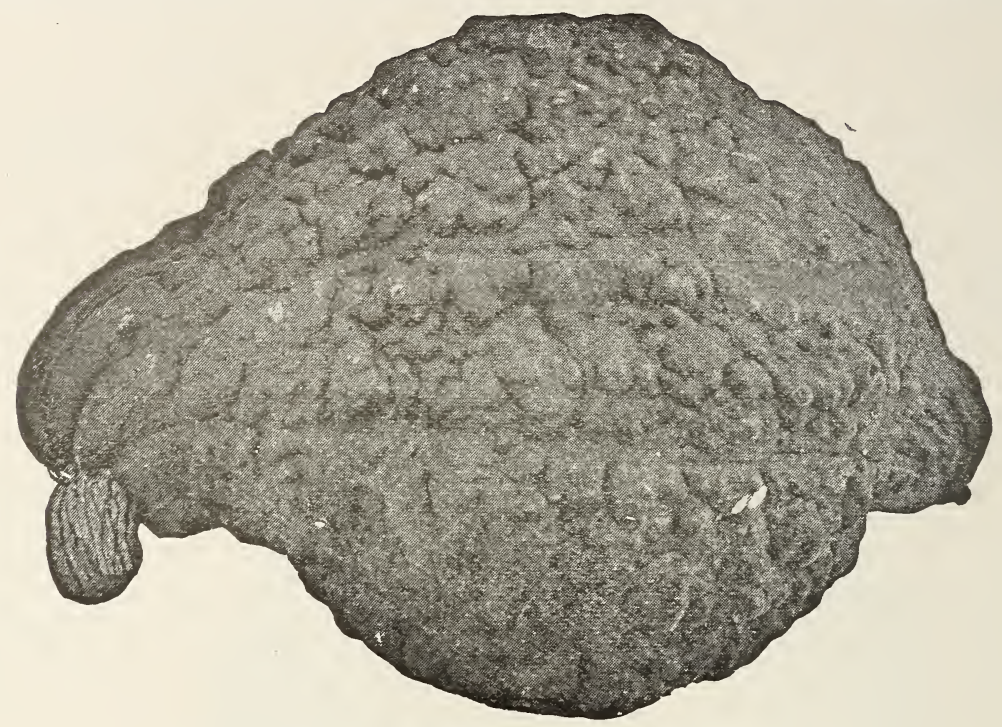

Squash. Chicago Warted Hubbard.

RHUBARB

$10 \mathrm{lbs}$.

Myatt's Victoria, the leading sort................\$0.80 \$0.75

\section{SALSIFY}

Mammoth Sandwich Island, the best variety . . . . . . . . $\$ 0.90 \$ 0.80$

\section{SPINACH}

Victoria, heavy, dark green foliage.............. $\$ 0.30$

Long Standing, remains long in good condition ............ .30

BLOOMSDALE OR SAVOY LEAVED, the best sort... . .30

Monstrous Viroflay Improved, thick leaved..............30

$\$ 0.25$

.25

.25

.25

\section{SQUASH}

Mammoth Chili, a good winter sort..............\$0.60

MAMMOTH WHITE BUSH, early and prolific........ .50

Golden Custard or Mammoth Yellow Bush ............ . .50

GIANT SUMMER CROOKNECK ............... .50

HUBBARD, the standard winter sort............. 60

CHICAGO VARTED finest strain of Hubbard......... .60

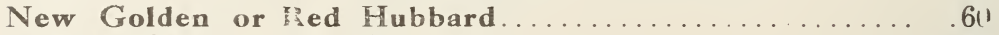

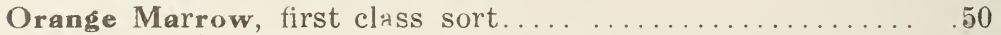

Fordhook, of splendid keeping quality. ...............5

White Summer Crookneck, fine market sort ............. . ()

\section{TOBACGO}

Per lb.

Connecticut Seed Leaf, best sort for North a d West. . . . . . . . \$2.50

White Burley, a popular variety ................... 


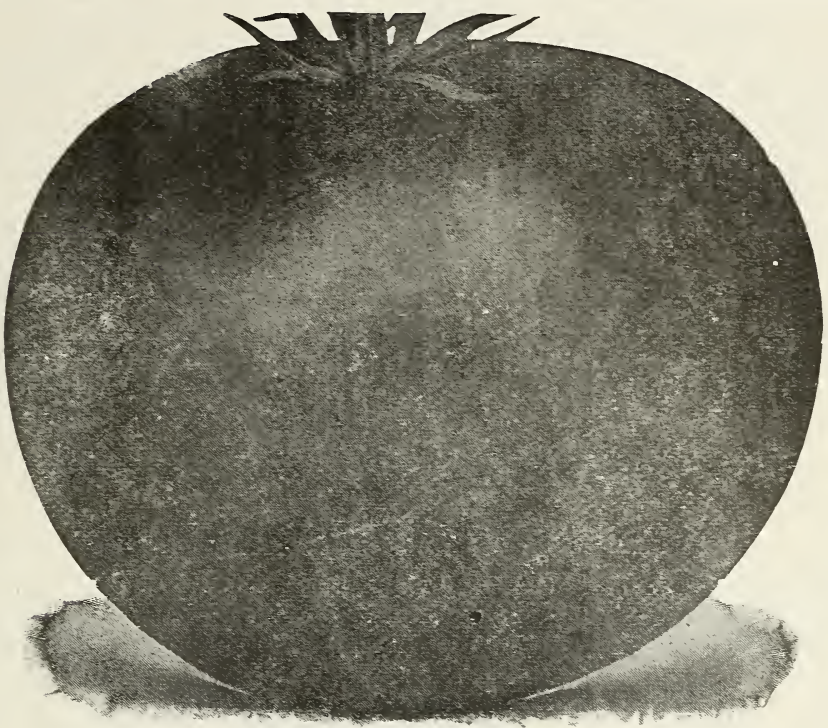

Tomato. Livingston's Beauty.

\section{TOMATO}

Per lb. Per lb.

ACME, early hardy, productive $\ldots \ldots \ldots \ldots \ldots \ldots \ldots \ldots \ldots 1.25 \quad \$ 1.10$

BEAUTY, leading sort. extra fine ..................... 1.25 1.00

STONE, excellent for canning ........................

Improved Trophy, large smooth, bright color............ $1.00 \quad .90$

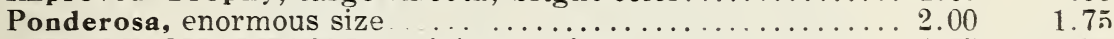

DWARF CHAMPION, upright growing ….............. $1.75 \quad 1.50$

Matchless, handsome sort, fine flavor.... ............. $1.25 \quad 1.10$

SPARK'S EARLIANA, remarkably early $\quad \ldots \ldots \ldots \ldots \ldots 1.50 \quad 1.35$

Pearshaped Red ......................... $1.50 \quad 1.30$

Pearshaped Yeliow................................ 1.50 1.30

Magnus. firm flesh, beautiful color ................... 1.50 1.35

New Dwarf Stone, uoright or tree form ............... 1.75 1.60

CHALK'S EARLY JEWEL, extra early, bright red....... 1.50 1.35

JUNE PINK, "earliest of all" ...................... 1.50 1.25

\section{TURNIP}

Purple Top Milan, extra early ..................... \$0.60

White Milan, tender, fine flavor ................... $\quad .60$

EXTRA EARLY WHITE FLAT DUTCH .......... .25

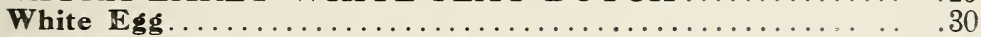

PURPLE TOP STRAP LEAF $\ldots \ldots \ldots \ldots \ldots \ldots \ldots \ldots$.

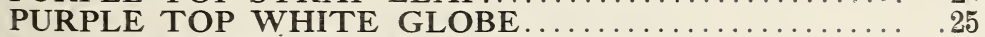

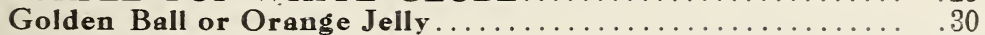

Leng White Cow Horn. heavy cropper.................. $\quad .30$

Amber Globe, large size......................... $\quad .30$ 


\section{HERBS}

\begin{tabular}{|c|c|}
\hline Sorrel Common... & \\
\hline 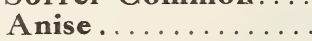 & \\
\hline$\ldots \ldots \ldots \ldots$ & \\
\hline Caraway ......... & \\
\hline$\ldots \ldots \ldots \ldots \ldots$ & \\
\hline Coriander ......... & \\
\hline Lavender . . . . . . . . & \\
\hline Sweet Marjoram.... & \\
\hline Sage, Broad Leaved & \\
\hline Summer Savory .... & .. \\
\hline Thyme $\ldots \ldots \ldots \ldots$ & \\
\hline
\end{tabular}

\section{GUMMED BAGS OR ENVELOPES}

For Retailing Small Seeds

Per 1000

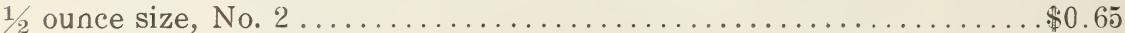

1 ounce size, No. 3

2 ounce size, No. 5.

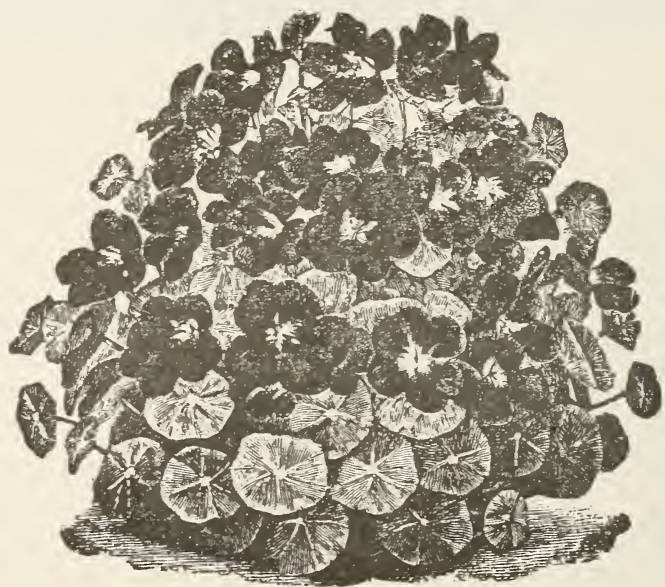

Nasturtium. Dwarf Mixed.

\section{NASTURTIUM}

Per lb.

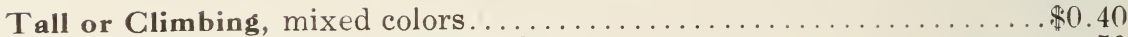

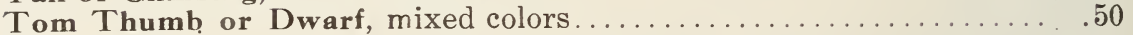

\section{SWEET PEAS}

Best Large Flowering Mixed, prepared from a well studied and care-

fully arranged formula and contains the newest and best varieties $\$ 0.45$

Fancy Spencer Mixed, a superb mixture of the famous Spencer or

Butterfly varieties, flowers heavily fringed ................ 75

Named Varieties, in separate colors ................... 60 


\section{VEGETABLE SEEDS IN PACKETS}

\section{(See Illustration Inside Back Cover Page)}

For the convenience of those who do not handle seeds in bulk, we can offer our Standard assortment of packet seeds. Our assortments are very complete, put up in handsome, well filled lithographed packets and well made, substantial case.

1000 Packet Assortment. ........................... \$15.00

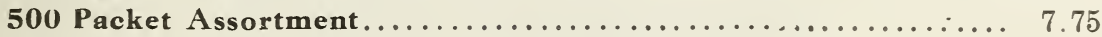

Retail value at $5 \mathrm{c}$ each, $\$ 50.00$ and $\$ 25.00$.

We replace at our expense all packages on which the vitality has expired, thus protecting you against any loss.

Self elevating display case included free with order.

\section{FLOWER SEEDS IN PACKETS}

Boxes containing the following assortment-all very finest varieties and ready sellers. Put up in an elegant self elevating display case.

$\begin{array}{rrl}5 & \text { packets } & \text { Aster, mixed } \\ 4 & 6 & \text { Sweet Alyssum } \\ 3 & \text { “ } & \text { Balsam } \\ 4 & \text { “ } & \text { Cypress Vine } \\ 4 & \text { “ } & \text { Marigold } \\ 10 & \text {. } & \text { Sweet Peas } \\ 18 & \text { ، } & \text { Nasturtiums } \\ 4 & \text { “ } & \text { Phlox } \\ 5 & \text { “ } & \text { Verbena } \\ 1 . & \therefore & \text { Pansy }\end{array}$

$\begin{array}{lll}4 & \text { packets } & \text { Poppy } \\ 5 & \text { ، } & \text { Petunia } \\ 3 & \text { ، } & \text { Bachelor's Button } \\ 3 & \text { “6 } & \text { Candytuit } \\ 3 & \text { ، } & \text { Cosmos } \\ 3 & \text { ‘ } & \text { Four-o’Clock } \\ 5 & \text { Morning Glory } \\ 3 & \text { ، } & \text { Zinnia } \\ 4 & \text { “ } & \text { Mignonette }\end{array}$

'Total, 100 packets - retail value $\$ 5.00$. Price with box ........... $\$ 2.50$

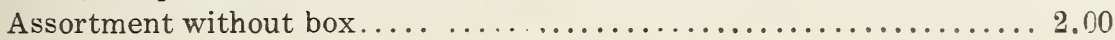

\section{GENT GARTONS}

Beans, Peas, Sweet Corn and Turnips have proven quick and profitable sellers. All put up in handsome packages to retail at $10 \mathrm{c}$ each.

\section{BEANS}

Kentucky Wonder, Stringless Green Pod, Early Red Valentine, Black

Wax, Golden Wax................................... 7c each

\section{CORN}

Early Minnesota, Early Evergreen, Stowell's Evergreen...... Price 5c each

\section{PEAS}

First and Best, Premium Gem, American Wonder, Everbearing, Large

White Marrowfat. .............................. 5 c each

\section{TURNIPS}

Purple Top Strap Leaf, Purple Top Globe ...............Price 5c each

Put up in large paper packets. 


\title{
AGRICULTURAL SEEDS
}

TERMS CASH

\author{
Weekly Price List Mailed Upon Application \\ All Prices Subject to Market Changes
}

\section{SELECTED SEED CORN}

Our stocks of Seed Corn are carefully grown, pure and true to name. The ears are well sorted, butted and tipped before shelling, so that all short round grains are removed. Perfectly cured and tested for germination. Samples on request.

\section{WHITE}

Per bu. shelled

Extra Early Adams, matures in about 80 days, small ears. ........ $\$ 1.50$ Silver Mine, early, good ears, yields good crops ...............2E Boone County White, our best main crop white corn; large thick ears

of perfect shape, deep grain........................... 1.25

Squaw Corn, blue and white ......................... 50

90 Day White, an extra early deep grained sort. Heavy yielder, fair

sized ears .....................................40

St. Charles White, red cob, white grain, large ears; a good yielder... 1.25

\section{STRIPED}

Improved Calico, main crop, large ear., good deep grain, a sure crop-

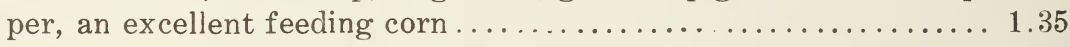

\section{RED}

90 Day Red or Bloody Butcher, very early, good sized ears........ 1.50

90 Day Yellow, very early, good size, good yielder............ 140

Reid's Yellow Dent, medium early, perfect ears, heavy yielder ..... 1.25

Jmproved Leaming, an ideal feeding corn, medium early, large ear,

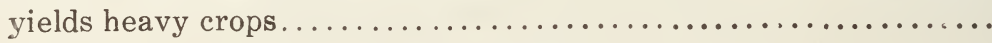

Golden Beauty, main crop, long ear, broad kernel.............. 1.30 No charge for bags on Seed Corn. SPECIAL PRICES IN CAR LOTS.

\section{POP CORN-For Popping}

White Rice

Shelled, 100 lbs.

White Pearl.

\section{BROOM GORN}

Grown especially for seed purposes, well ripened and tested.

Standard Evergreen 


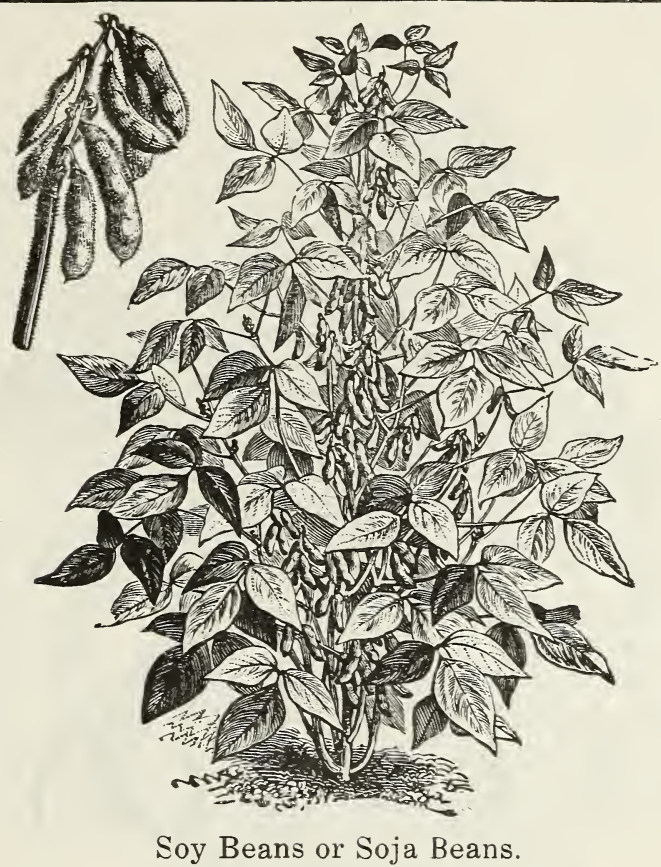

FIELD PEAS AND BEANS Per bu. Soy Beans, Early Yellow ...........................\$3.00 Soy Beans, Late Mammoth ............................ 20 Cow Peas, New Era.......

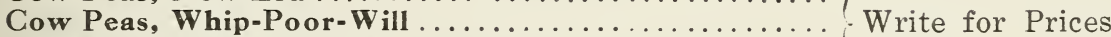
White Canada Field Peas

\section{SORGHUMS}

Coleman's

Early Amber.

Early Orange............................ Per

\section{KAFFIR CORN}

Red, for seed..........

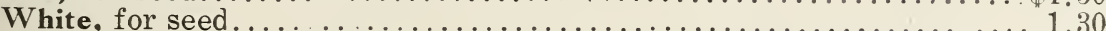




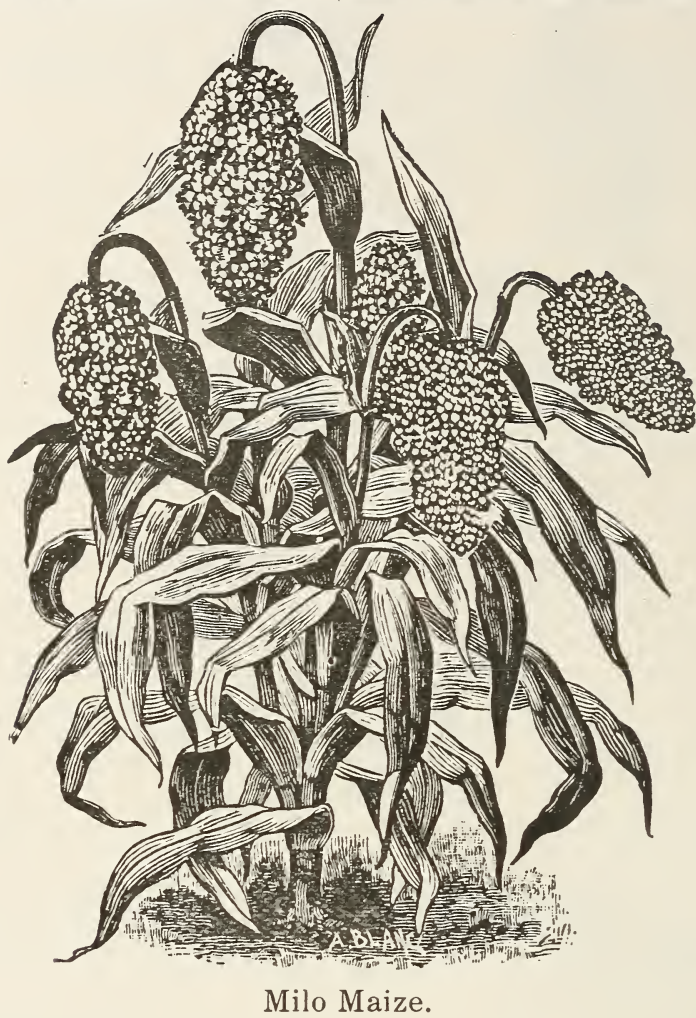

MILO MAIZE

Common, choice

Common, prime.

German, fancy, true rounded seeded

German, choice

German, prime.

Siberian Millet, choice.....

Hungarian, choice......

Japanese or Barnyard Millet

Pearl or Cat-tail

Choice, recleaned ..

\section{JERUSALEM CORN}

Per lb. 100 lbs. ...... $\$ 0.04$

\section{RAPE-DWARF ESSEX}

We use great care to have our stock of this the true dwarf biennial variety. We import from only reliable dealers and are confident our stock is the very best obtainable. Cheap rape should be avoided. Per lb. $100 \mathrm{lbs}$. True Dwarf Essex . $\$ 0: 09 \quad \$ 750$ 


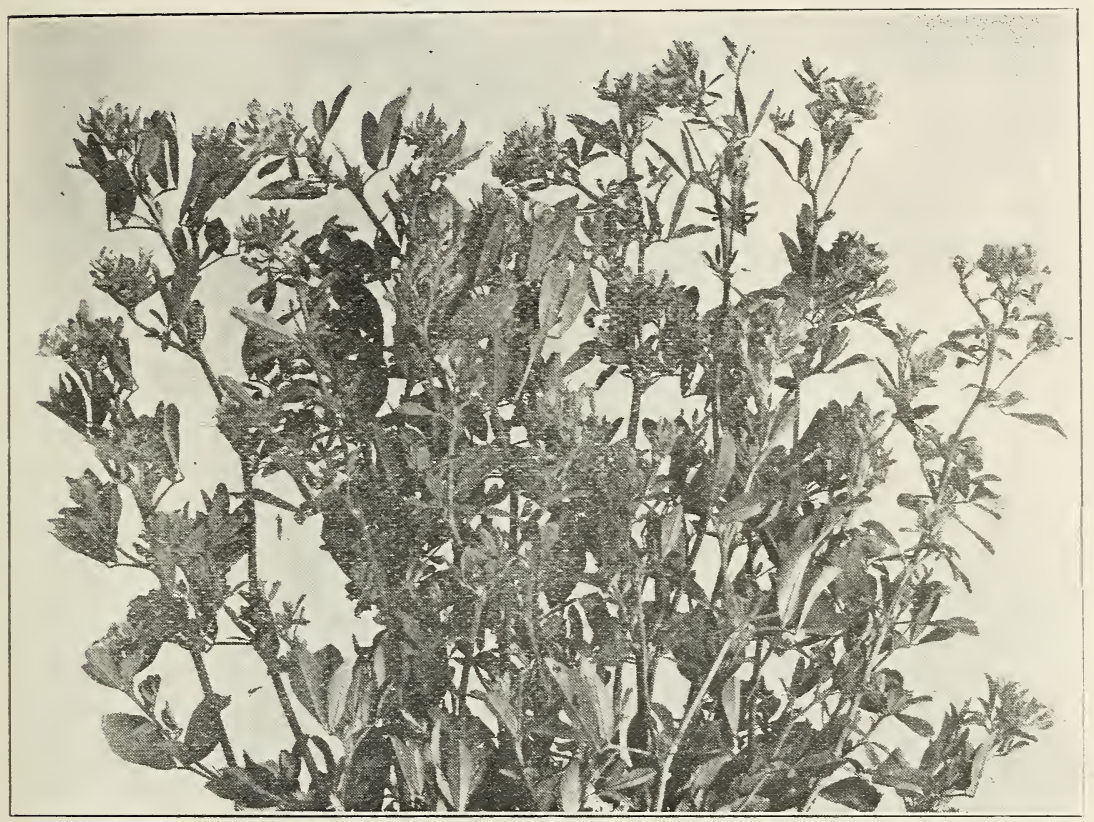

Alfalfa.

\section{CLOVERS}

Per 100 lbs.

Red or Medium, fair ...................... \$15.00

Red or Medium, prime .................... 17.00

$\$ 16.00$

Red or Medium, choice ....................... 1850

15.50

Red or Medium, fancy...................... 19.50

Red or Medium, STANDARD BRAND ............

Mammoth, prime ........................... 17.50

Mammoth. choice............................. 19.00

Mammoth, fancy ..... ........ .. $\quad \ldots \ldots \ldots . \quad 20.00$

Alfalfa, fair ............................. 13.00

Alfalfa, prime........................... 15.50

19.00

20.00

21.00

18.00

19.50

20.50

13.50

16.00

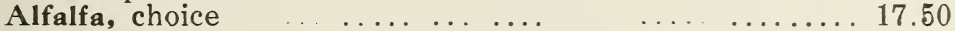

18.00

Alfalfa, fancy.......................... 19.00

19.50

Alfalfa, STANDARD BRAND. ... ............ 20.50

Alfalfa, Turkestan.

Alsike, prime....... ...................... 1500

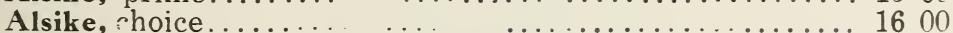

Alsike, fancy .... ............................. 17. (1

White, prime .......................... 4300

White, choice .......................... 4600

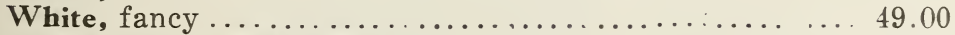

Sweet Clover, White Bokhara, choice ........... . 15.50

Sweet Clover, White Bokhara, fancy.............. 17.00

Sweet Clover, Yellow Biennial, choice ..............

Prices Fluctuate. Samples Mailed on Request. 


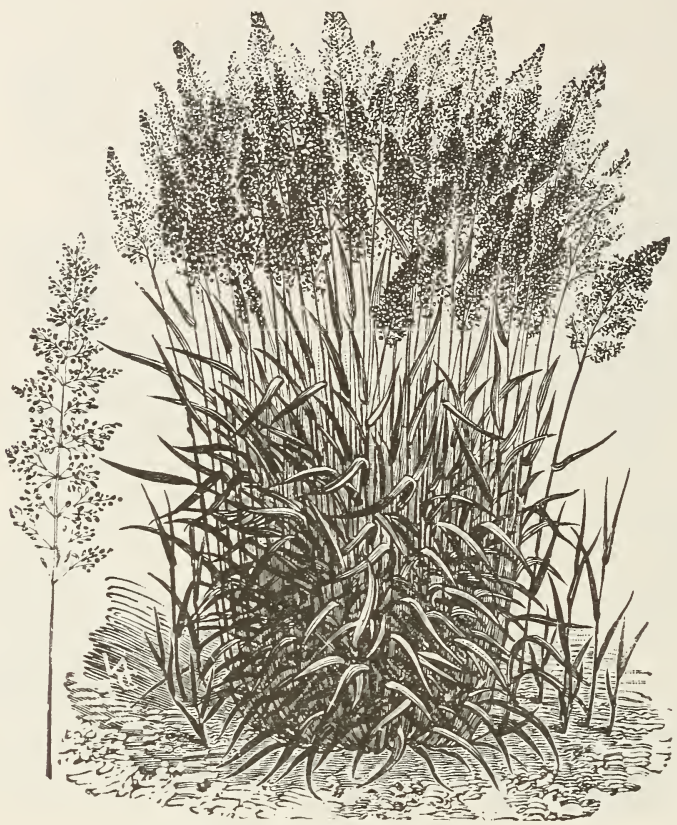

Red Top or Herds Grass.

GRASS SEEDS

Per 100 lbs.

Timothy, fair $\$ 6.50$

Timothy, prime ............................. 7.50

Timothy, choice .......................... 8.00

Timothy, fancy

8.50

8.25

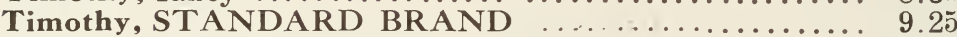

Kentucky Blue Grass, choice.................. 8.00

Kentucky Blue Grass, fancy .................... 9.75

Kentucky Blue Grass, STANDARD BRAND ........ 1100

Red Top, Solid Seed, fancy . . . . . . . . . . . . . . . . 13.50

Red Top, Solid Seed, STANDARD BRAND ......... 15.00

Ry• Grass, English or Perennial, choice ............ 7.00

8.75

English Blue Grass or Meadow Fescue, choice ......... 800

English Blue Grass or Meadow Fescue, fancy.... . . . . . . 9.50

Orchard Grass, prime ................... 1450

Orchard Grass, choice .... . .............. 15.50

Orchard Grass, fancy . . . . . . . . . . . . . . . . 16.50

Bromus Inermis, choice . . . . . . . . . . . . . . . . 10.50

Mixed Lawn Grass, STANDARD BRAND ........... 10.50

A mixture of several suitable grasses, producing a fine green sward in six or seven weeks from sowing. Highly recommended.

Sudan Grass............................ 7.00 7.50

Bermuda Grass..................................... 0.35 

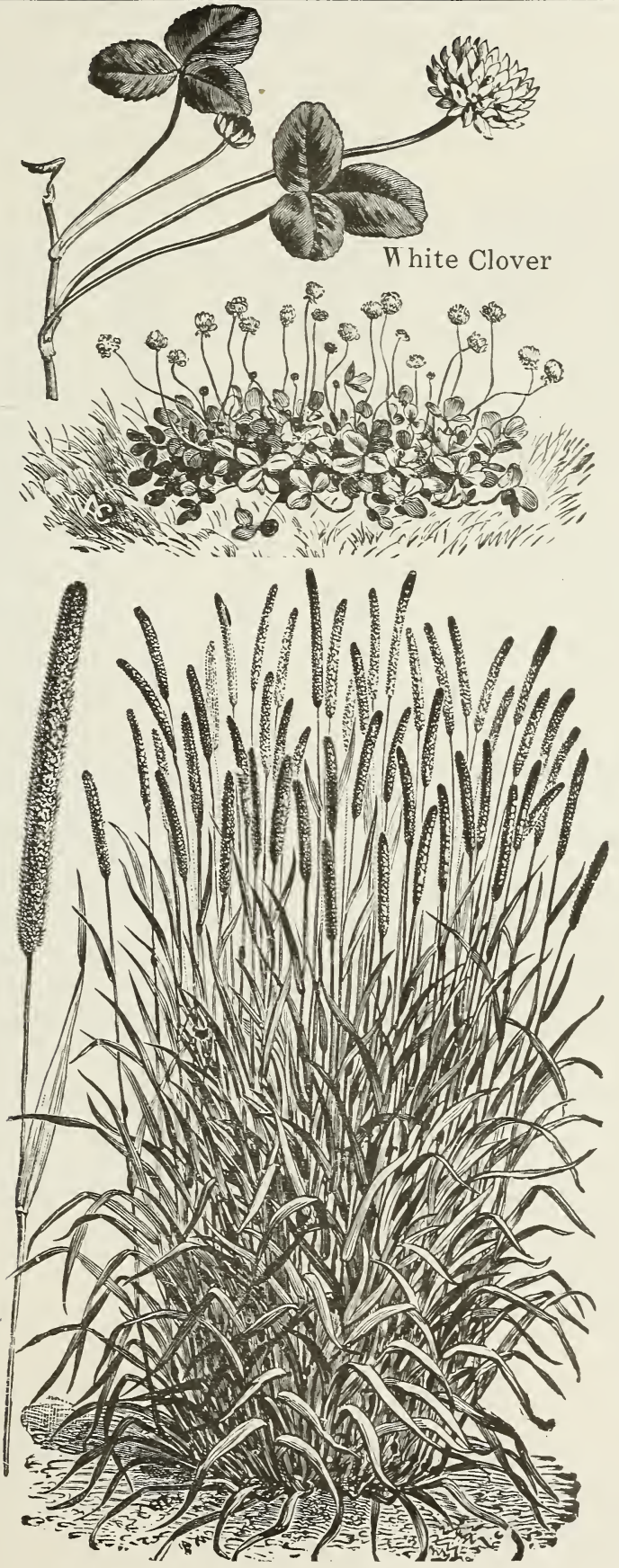

Timothy 
OIL MEAL

Linseed Meal. Old rrucess.....................\$2.15 $\$ 40.50$

Per $100 \mathrm{lbs}$. Per ton
$\ldots \ldots 2.15$

\section{Prices Subject to Market Change.}

\section{POULTRY SUPPLIES}

Genume Oyster Shell, fine or coarse ground............. \$ \$0.70

Genuime Oyster Shell, j bag lots .................... 65

Crushed Poultry Shell, fine or coarse ground............... 55

Crushed Poultry Shell, 5 bag lots. ....................... ji

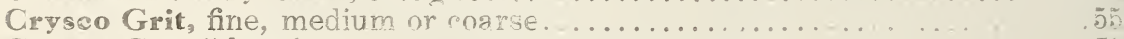

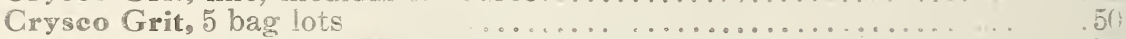

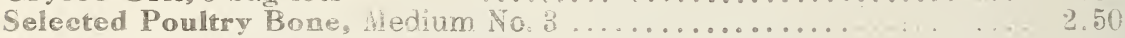

Selected Poultry Bone, Coarse \$o. 2 . . . . . . . . . . . . 5 ?

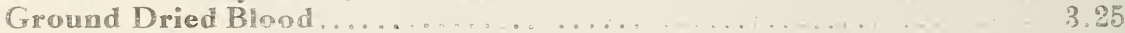

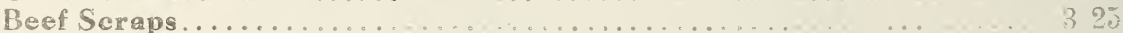

Chick Food, Imperial Brand. Vo grit or shell...... . . . . . $\quad 1.75$

Chiok Food, Challenge Brand. No grit or shell....... 1.6

Laying Food, Reliahle Brand. No grit or shell............. i.6.

Subjece ro Mrirket Changes.

Ast for Srecinl Prices in Large Quantities.

\section{ONION SETS}

These are ore of our specialties. We grow annually a large acreage and handle many thousand bushels in adurion. All sets we send out are sound, dry anu unsprouted. They are csirefully screened and fanned and of a uni form size.

Note-Bags are weighed in free. Barrels holding : $31_{2}$ busiels, 3nceach. Customers will please state packages desired.

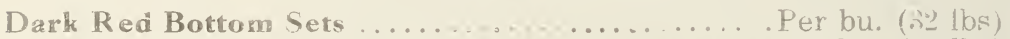

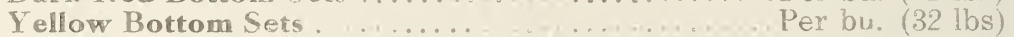

White Bottom Sets ... .................. Per bu. (32 ibs)

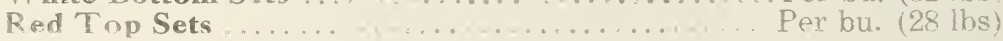

Write

for

Prices

Above Prices for Feloruary Shipment Only.

March Delivery 10c Higher.

\section{PIIRD SEEDS}

Hernp

Per lb. 100 lbs.

Camary

$\$ 05 \frac{1}{2}$

Rape

08

6. 00

Sunfliower

(1) 6

7.50

Millet

Mixed Bird Seed

.74

ว. 50

06

3.50

$+75$

\section{TREL SELDS}

Black or Yellow Locust $\$ 0.25$ 


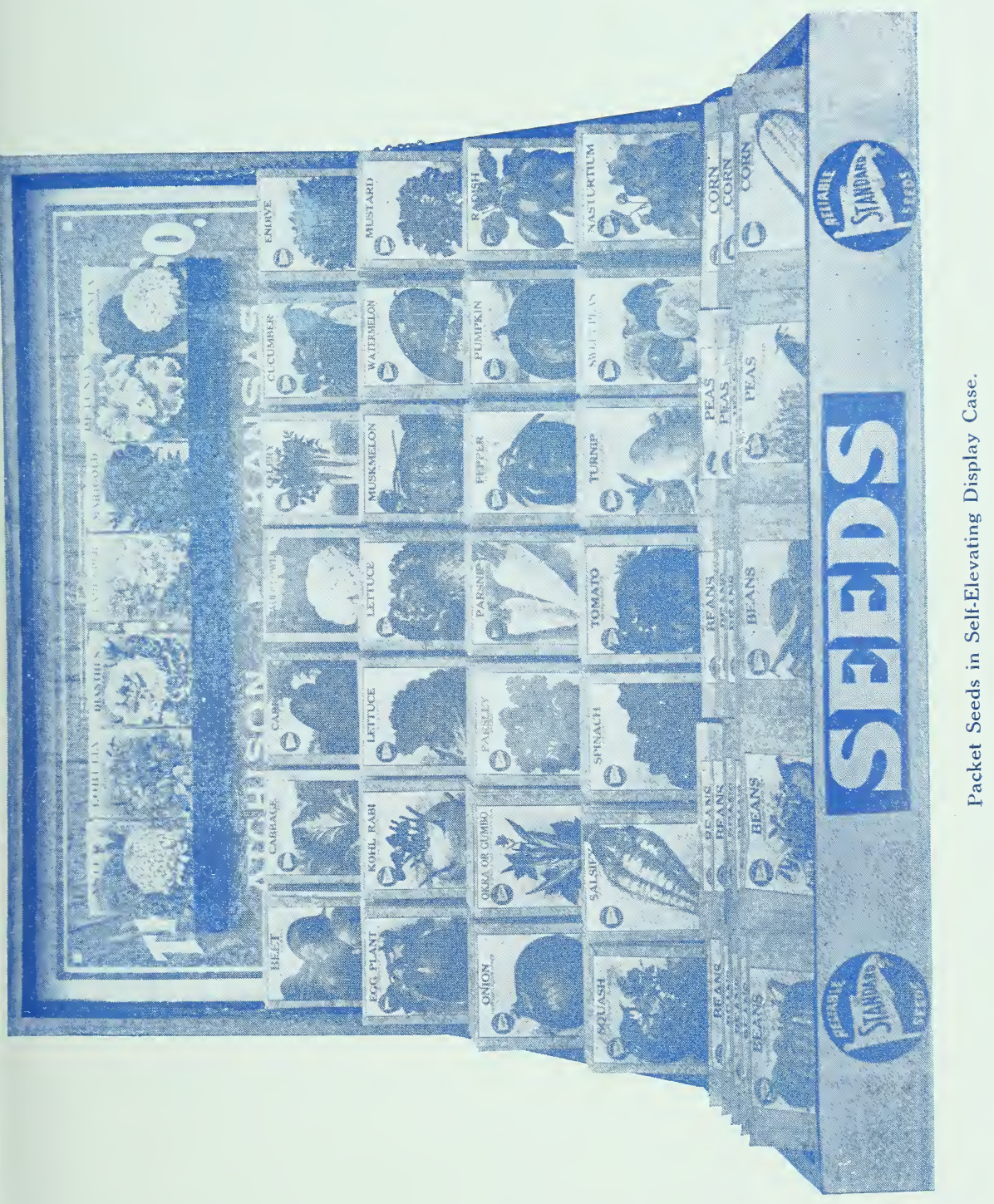




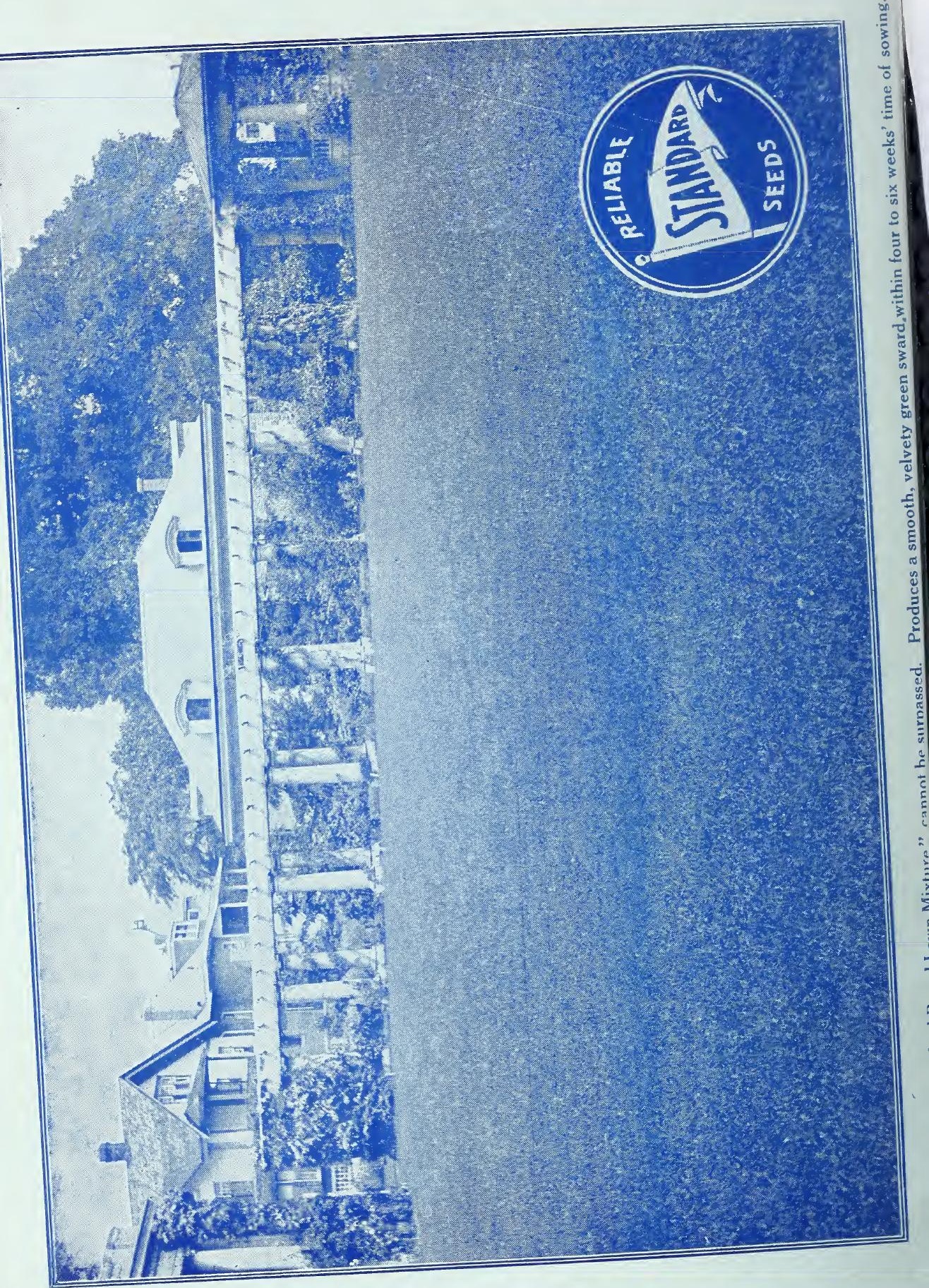

